\title{
Chlorine Content of Mother's Urine and Their Arakawa's Reaction.
}

136th Report of the Peroxidase Reaction. (83rd Human Milk Study.)

\author{
By \\ Haruka Umemura. \\ (梅 村 晴 香) \\ (From the Department of Pediatrics, Faculty of Medicine, \\ Tohoku Imperial University, Sendai. \\ Director: Prof. A. Sato.)
}

\section{Introduction.}

In 1934 it was published by Nozaki) (Cf. 46th Report of the Peroxidase Reaction) from this Laboratory as a surprising and unexpected fact that human milk positive to Arakawa's reaction* is a chlorine-poor milk, and that Arakawa-negative milk is a chlorine-rich milk. This assertion has lately been confirmed by Ishii (Cf. 87th Report of the Peroxidase Reaction), ${ }^{2)}$ who made a further investigation into the relation in a much larger number of materials. Now I thought, if the amount of milk chlorine is considerably different according as lactating mothers are Arakawa-positive or -negative, then what would become of that surplus of the salt corresponding to the difference? Of course they must be probably taking an approximately equal amount of chlorine, for it is utterly impossible that mothers with milk positive to Arakawa's reaction are daily taking much more table salt than

1) J. Nozaki, Tóhoku J. Exp. Med., 1934, 23, 60.

* Arakawa-positive may be used in two different senses. One of these is: Arakawa-positive in a biochemical sense. A sample of human milk is said to have become Arakawa-pesitive, when it becomes blue on the addition of Arak a w a's reagent. There it means that the sample is not negative to Arakawa's reaction. Arakawapositive in a clinical sense: A sample of human milk is clinically Arakawa-positive only when it shows such a reaction as + or $H$ in one minute of the addition of A ra$\mathrm{k}$ a wa's reagent. Another sample of human milk may be Arakawa-positive in the first described sense, but yet clinically negative.

a) M. Is hi i, Tohoku J. Exp. Med., 1937, 31, 580. 
Arakawa-negative mothers. So I wanted to investigate the relation between chlorine content of mothers' urine and their Arakawa's reaction.

\section{Experimental Method.}

The method which has generally been used for the volumetric micro determination of chlorine in biological fluids in our Laboratory is that of Rusznyak ${ }^{3)}$ for blood chlorine. I applied, for reasons of economy, the micro method to human urine using 0.1 c.c., and the following application of the method of Rusznyak was found to be satisfactory for my purpose.

The principle is the oxydation of the organic substances in urine with potassium permanganate, and then the precipitation of the chlorides by a standard solution of silver nitrate and the subsequent titration of the excess silver nitrate by ammonium thiocyanate, using iron alum as an indicator. The end point is the first permanent red tint due to the formation of ferricyanate.

\section{Procedure.}

In a 20 c.c. volumetric flask place 0.1 c.c. of urine accurately measured, 4 c.c. of $0.01 \mathrm{~N}$ silver nitrate solution, 1 c.c. of concentrated nitric acid. Boil gently with a drop of potassium permanganate solution $1 \mathrm{~N}$ until it becomes tinged with rust colour, and add a small amount of pure grape sugar powder until the colour disappears. Cool the flask in running water or at room temperature and titrate the excess silver nitrate with $0.01 \mathrm{~N}$ ammonium thiocyanate solution, adding a few drops of saturated iron alum as an indicator.

\section{Determination.}

Substract the number of c.c. of ammonium thiocyanate used in the titration from the number of c.c. of $0.01 \mathrm{~N}$ silver nitrate solution; the difference is the volume of the silver solution used to precipitate the chlorine in 0.1 c.c. of the urine examined. Since 1 c.c. of the silver nitrate solution-is equivalent to 0.00035 grms. of chlorine, the calculation is as follows :-

$\frac{(\mathrm{a}-\mathrm{b}) \times 0.000355}{0.1} \times 100=$ chlorine (grms.) in 100 c.c. of urine

$a=$ the number of c.c. of silver nitrate solution.

$\mathrm{b}=$ the number of c.c. of ammonium thiocyanate.

3) St. Rusznyak, Biochem. Z., 1921, 114, 23. 
Accuracy.

To test the accuracy of the method, determinations were made of the chlorine content of the urine in a number of cases. Then a theoretical solution of sodium chloride was added and determinations were performed again. The figures are given below (Cf. Tables 1 and 2):-

TABLE 1.

Recovery of $\mathrm{NaCl}$ added to human urine.

\begin{tabular}{|c|c|c|c|c|}
\hline 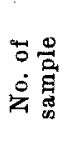 & 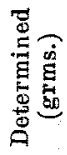 & 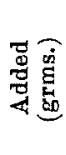 & 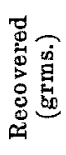 & 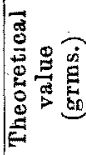 \\
\hline 1 & 1.26 & 0.100 & 1.36 & 1.36 \\
\hline 2 & 0. & 0.100 & .0 .88 & 0.87 \\
\hline 3 & 1.53 & 0.1 & 1.64 & 1.63 \\
\hline 4 & 1.06 & 0.100 & 1.16 & 1.16 \\
\hline 5 & 1.42 & 0.1 & 1.52 & 1.52 \\
\hline 6 & 0.88 & 0.100 & 0.98 & 0.99 \\
\hline
\end{tabular}

TABLE 2.

Comparison of results obtained with Volhard-Salkowski's and author's method.

\begin{tabular}{c|c|c}
\hline $\begin{array}{c}\text { No. of } \\
\text { sample }\end{array}$ & $\begin{array}{c}\text { Volhard- } \\
\text { Salkows ik's } \\
\text { method } \\
\text { (grms.) }\end{array}$ & $\begin{array}{c}\text { Author's } \\
\text { method } \\
\text { (grms.) }\end{array}$ \\
\hline 1 & 1.04 & 1.02 \\
2 & 0.61 & 0.59 \\
3 & 1.45 & 1.44 \\
4 & 1.45 & 1.40
\end{tabular}

As shown in Tables 1 and 2, the present method is not so exact as the macro method, but it is sufficiently accurate for the present purpose (see later).

\section{Materials.}

All the 202 urine samples were mostly from mothers of outpatients of our Department (Cf. Table 3). Cases of mastitis, mammary tumours and cases with menstruation just at the time of examination were excluded. The urine was obtained in the morning (9-10 a.m.). At the same time mother's milk was axamined with Arakawa's reagent in each case. Cases within one month after the delivery were discarded from this paper.

\section{Result of Experiment.}

As is seen from Table 3 (Cf. Table 3), chlorine content in urine samples from mothers is subject to a very remarkable variation, but it is interesting to find in Tables 5 and 6 (Cf. Tables 5 and 6 ) that the samples of Arakawa-negative mothers are the poorest in chlorine content $(0.70$ grms. per 100 c.c. on an average). Samples of intermediate reactions show intermediate values $(0.882,0.893,0.990,0.820,0.885$, 
Table 3.

Chlorine content of urine of lactating mothers.

\begin{tabular}{|c|c|c|c|c|c|}
\hline 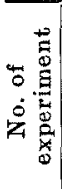 & 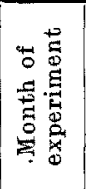 & 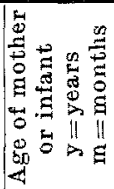 & 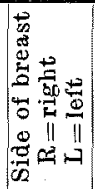 & 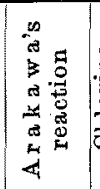 & 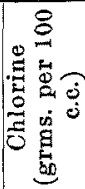 \\
\hline 1 & $\begin{array}{c}\mathrm{X} \\
(1936)\end{array}$ & $\begin{array}{l}28 \mathrm{y} \\
17 \mathrm{~m} \\
\end{array}$ & $\begin{array}{l}\mathrm{R} \\
\mathbf{L}\end{array}$ & $\begin{array}{l}1(++) \\
1(+)\end{array}$ & 0.91 \\
\hline 2 & $\Rightarrow$ & $\begin{array}{r}30 \mathrm{y} \\
8 \mathrm{~m}\end{array}$ & $\begin{array}{l}R \\
\mathrm{~L}\end{array}$ & $\begin{array}{l}6( \pm) \\
2(+t)\end{array}$ & 0.86 \\
\hline 3 & $\Rightarrow$ & $\begin{array}{r}19 \mathrm{y} \\
6 \mathrm{~m}\end{array}$ & $\begin{array}{l}\mathrm{R} \\
\mathrm{L}\end{array}$ & $\begin{array}{l}1(+) \\
4(-)\end{array}$ & 0.93 \\
\hline 4 & $\pi$ & $\begin{array}{r}27 \mathrm{~g} \\
2 \mathrm{~m} \\
\end{array}$ & $\begin{array}{l}\mathrm{R} \\
\mathrm{L}\end{array}$ & $\begin{array}{l}7( \pm) \\
7( \pm)\end{array}$ & 0.62 \\
\hline 5 & n & $\begin{array}{l}25 \mathrm{y} \\
12 \mathrm{~m}\end{array}$ & $\begin{array}{l}\mathrm{R} \\
\mathrm{L}\end{array}$ & $\left|\begin{array}{r}16(-) \\
1( \pm)\end{array}\right|$ & 0.71 \\
\hline 6 & $n$ & $\begin{array}{l}25 \mathrm{y} \\
18 \mathrm{~m}\end{array}$ & $\begin{array}{l}\mathrm{R} \\
\mathrm{L}\end{array}$ & $\begin{array}{r}7( \pm) \\
16(-)\end{array}$ & 0.89 \\
\hline 7 & $"$ & $\begin{array}{l}19 \mathrm{y} \\
11 \mathrm{~m}\end{array}$ & $\begin{array}{l}\mathrm{R} \\
\mathrm{L}\end{array}$ & $\left|\begin{array}{l}4(+) \\
5(+)\end{array}\right|$ & 0.78 \\
\hline 8 & $\eta$ & $\begin{array}{r}25 \mathrm{y} \\
2 \mathrm{~m}\end{array}$ & $\begin{array}{l}\mathrm{K} \\
\mathrm{L}\end{array}$ & $\left|\begin{array}{r}10(-) \\
6( \pm)\end{array}\right|$ & 1.04 \\
\hline 9 & $\pi$ & $\begin{array}{r}36 y \\
8 m \\
\end{array}$ & $\begin{array}{l}\mathrm{R} \\
\mathrm{L}\end{array}$ & $\left|\begin{array}{l}10(-) \\
10(-)\end{array}\right|$ & 1.00 \\
\hline 10 & ग & $\begin{array}{r}32 \mathrm{y} \\
2 \mathrm{~m} \\
\end{array}$ & $\begin{array}{l}\mathrm{R} \\
\mathrm{L}\end{array}$ & $\left|\begin{array}{l}7( \pm) \\
7( \pm)\end{array}\right|$ & 0.43 \\
\hline 11 & $"$ & $\begin{array}{l}27 \mathrm{y} \\
11 \mathrm{~m}\end{array}$ & $\begin{array}{l}\mathrm{R} \\
\mathrm{L}\end{array}$ & $\begin{array}{l}16(-) \\
16(-)\end{array}$ & 0.50 \\
\hline 12 & " & $\begin{array}{l}29 \mathrm{y} \\
14 \mathrm{~m}\end{array}$ & $\begin{array}{l}\mathrm{R} \\
\mathrm{L}\end{array}$ & $\left|\begin{array}{l}4(-) \\
4(-)\end{array}\right|$ & 0.77 \\
\hline 13 & $\pi$ & $\begin{array}{r}25 \mathrm{y} \\
7 \mathrm{~m} \\
\end{array}$ & $\begin{array}{l}\mathrm{R} \\
\mathrm{L} \\
\end{array}$ & $\begin{array}{l}12(-) \\
16(-)\end{array}$ & 1.06 \\
\hline 14 & $n$ & $\begin{array}{l}38 \mathrm{y} \\
18 \mathrm{~m}\end{array}$ & $\begin{array}{l}\mathrm{R} \\
\mathrm{L}\end{array}$ & $\left|\begin{array}{c}11(-) \\
3(-)\end{array}\right|$ & 0.89 \\
\hline 15 & $n$ & $\begin{array}{r}25 \mathrm{y} \\
2 \mathrm{~m} \\
\end{array}$ & $\begin{array}{l}\mathbf{R} \\
\mathrm{L}\end{array}$ & $\begin{array}{l}16(-) \\
16(-)\end{array}$ & 0.71 \\
\hline 16 & $n$ & $\begin{array}{r}20 \mathrm{y} \\
9 \mathrm{~m} \\
\end{array}$ & $\begin{array}{l}\mathbf{R} \\
\mathrm{L}\end{array}$ & $\begin{array}{l}2(+) \\
3(+)\end{array}$ & 1.32 \\
\hline 17 & $n$ & $\begin{array}{r}25 \mathrm{y} \\
9 \mathrm{~m} \\
\end{array}$ & $\begin{array}{l}\mathrm{R} \\
\mathrm{L}\end{array}$ & $\begin{array}{l}14(-) \\
16(-)\end{array}$ & 0.67 \\
\hline 18 & $n$ & $\begin{array}{r}26 \mathrm{y} \\
2 \mathrm{~m}\end{array}$ & $\begin{array}{l}\mathbf{R} \\
\mathrm{L}\end{array}$ & $\begin{array}{l}7(+) \\
3( \pm)\end{array}$ & 0.99 \\
\hline 19 & $n$ & $\begin{array}{r}23 \mathrm{y} \\
1 \mathrm{~m}\end{array}$ & $\begin{array}{l}\mathbf{R} \\
\mathrm{L}\end{array}$ & $\begin{array}{l}2(-) \\
2(-)\end{array}$ & 0.78 \\
\hline 20 & $n$ & $\begin{array}{l}26 \mathrm{y} \\
14 \mathrm{~m}\end{array}$ & $\begin{array}{l}\mathbf{R} \\
\mathbf{L}\end{array}$ & $\begin{array}{r}2(-) \\
16(-)\end{array}$ & 0.68 \\
\hline 21 & $n$ & $\begin{array}{l}38 \mathrm{~g} \\
13 \mathrm{~m} \\
\end{array}$ & $\begin{array}{l}\mathbf{R} \\
\mathbf{L} \\
\end{array}$ & $\begin{array}{l}3(+) \mid \\
3(+)\end{array}$ & 0.89 \\
\hline 22 & $n$ & $\begin{array}{r}39 \mathrm{y} \\
5 \mathrm{~m} \\
\end{array}$ & $\begin{array}{l}\mathbf{R} \\
\mathbf{L} \\
\end{array}$ & $\left|\begin{array}{l}9(+) \\
9( \pm)\end{array}\right|$ & 0.96 \\
\hline 23 & $\because$ & $\begin{array}{r}27 \mathrm{y} \\
6 \mathrm{~m}\end{array}$ & $\begin{array}{l}\mathrm{R} \\
\mathrm{L}\end{array}$ & $\left|\begin{array}{l}10(-) \\
14(-)\end{array}\right|$ & 0.79 \\
\hline
\end{tabular}

\begin{tabular}{|c|c|c|c|c|c|}
\hline 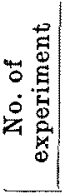 & 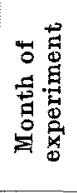 & 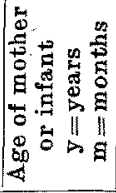 & 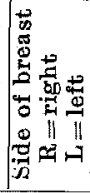 & 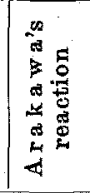 & 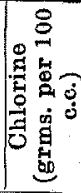 \\
\hline 24 & $n$ & $\begin{array}{l}21 \mathrm{y} \\
23 \mathrm{~m}\end{array}$ & $\begin{array}{l}\mathbf{R} \\
\mathrm{L} \\
\end{array}$ & $\begin{array}{r}7( \pm) \\
12(-)\end{array}$ & 0.80 \\
\hline 25 & 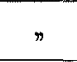 & $\begin{array}{r}30 \mathrm{y} \\
4 \mathrm{~m}\end{array}$ & $\begin{array}{l}\mathrm{R} \\
\mathrm{L}\end{array}$ & $\begin{array}{l}1(+) \\
3(+)\end{array}$ & 0.99 \\
\hline 26 & $n$ & $\begin{array}{r}25 \mathrm{y} \\
5 \mathrm{~m}\end{array}$ & $\begin{array}{l}\mathbf{R} \\
\mathbf{L}\end{array}$ & $\begin{array}{l}1(H) \\
1(H)\end{array}$ & 0.89 \\
\hline 27 & $n$ & $\begin{array}{l}29 \mathrm{~m} \\
14 \mathrm{~m} \\
\end{array}$ & $\begin{array}{l}\mathrm{R} \\
\mathrm{L}\end{array}$ & $\left(\begin{array}{r}11(-) \\
3(-)\end{array}\right.$ & 0.82 \\
\hline 28 & $"$ & $\begin{array}{r}27 \mathrm{y} \\
3 \mathrm{~m}\end{array}$ & $\begin{array}{l}\mathrm{R} \\
\mathrm{L}\end{array}$ & {$\left[\begin{array}{c}11( \pm) \\
9( \pm)\end{array}\right.$} & 0.92 \\
\hline 29 & $n$ & $\begin{array}{l}31 \mathrm{y} \\
14 \mathrm{~m}\end{array}$ & $\begin{array}{l}\mathbf{R} \\
\mathrm{L}\end{array}$ & $\begin{array}{l}1(+) \\
7(-)\end{array}$ & 0.79 \\
\hline 30 & $"$ & $\begin{array}{r}31 \mathrm{y} \\
6 \mathrm{~m}\end{array}$ & $\begin{array}{l}\mathbf{R} \\
\mathbf{L}\end{array}$ & $\mid \begin{array}{l}1(+) \\
2(+)\end{array}$ & 1.08 \\
\hline 31 & $\pi$ & $\begin{array}{r}31 \mathrm{y} \\
1 \mathrm{~m} \\
\end{array}$ & $\begin{array}{l}\mathbf{R} \\
\mathbf{L}\end{array}$ & $\begin{array}{l}16(-) \\
15(-)\end{array}$ & 0.48 \\
\hline 32 & $\pi$ & $\begin{array}{l}23 \mathrm{y} \\
13 \mathrm{~m} \\
\end{array}$ & $\begin{array}{l}\mathbf{R} \\
\mathbf{L}\end{array}$ & $\begin{array}{l}15(-) \\
15(-)\end{array}$ & 1.08 \\
\hline 33 & $n$ & $\begin{array}{l}28 \mathrm{y} \\
20 \mathrm{~m}\end{array}$ & $\begin{array}{l}\mathbf{R} \\
\mathrm{L}\end{array}$ & $\begin{array}{l}11( \pm) \\
11( \pm)\end{array}$ & 0.89 \\
\hline 34 & $n$ & $\begin{array}{r}25 \mathrm{y} \\
4 \mathrm{~m} \\
\end{array}$ & ${ }_{\mathbf{L}}^{\mathbf{R}}$ & $\begin{array}{l}16(-) \\
16(-)\end{array}$ & 0.74 \\
\hline 35 & $\Rightarrow$ & $\begin{array}{l}24 \mathrm{y} \\
17 \mathrm{~m} \\
\end{array}$ & $\begin{array}{l}\mathbf{R} \\
\mathbf{L} \\
\end{array}$ & $\begin{array}{l}4(+) \\
4(+)\end{array}$ & 1.21 \\
\hline 36 & $"$ & $\begin{array}{l}21 \mathrm{y} \\
12 \mathrm{~m}\end{array}$ & $\begin{array}{l}\mathbf{R} \\
\mathbf{L} \\
\end{array}$ & $\begin{array}{l}4(+) \\
4(+)\end{array}$ & 1.17 \\
\hline 37 & $n$ & $\begin{array}{r}20 \mathrm{~g} \\
4 \mathrm{~m} \\
\end{array}$ & $\begin{array}{l}\mathbf{R} \\
\mathbf{L}\end{array}$ & $\begin{array}{l}9( \pm) \\
5( \pm)\end{array}$ & 0.39 \\
\hline 38 & 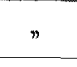 & $\begin{array}{r}28 \mathrm{y} \\
1 \mathrm{~m} \\
\end{array}$ & $\begin{array}{l}\mathbf{R} \\
\mathrm{L} \\
\end{array}$ & $\begin{array}{c}* \\
5( \pm)\end{array}$ & 0.84 \\
\hline 39 & $n$ & $\begin{array}{r}33 \mathrm{y} \\
2 \mathrm{~m} \\
\end{array}$ & $\begin{array}{l}\mathrm{R} \\
\mathrm{L}\end{array}$ & $16(-)$ & 0.53 \\
\hline 40 & $n$ & $\begin{array}{r}22 \mathrm{y} \\
4 \mathrm{~m} \\
\end{array}$ & $\begin{array}{l}\mathbf{R} \\
\mathbf{L}\end{array}$ & $\begin{array}{l}5(+) \\
5(H)\end{array}$ & 0.97 \\
\hline 41 & $n$ & $\begin{array}{r}28 \mathrm{y} \\
2 \mathrm{~m} \\
\end{array}$ & $\begin{array}{l}\mathrm{R} \\
\mathrm{L}\end{array}$ & $\left|\begin{array}{l}13(-) \\
13(-)\end{array}\right|$ & 0.92 \\
\hline 42 & $n$ & $\begin{array}{r}25 \mathrm{y} \\
2 \mathrm{~m} \\
\end{array}$ & $\begin{array}{l}\mathrm{R} \\
\mathrm{L} \\
\end{array}$ & $\left|\begin{array}{c}2(-) \\
16(-)\end{array}\right|$ & 0.57 \\
\hline 43 & $n$ & $\begin{array}{l}27 \mathrm{y} \\
12 \mathrm{~m} \\
\end{array}$ & $\begin{array}{l}\mathrm{R} \\
\mathrm{L}\end{array}$ & $\left|\begin{array}{l}7(+) \\
5( \pm)\end{array}\right|$ & 0.92 \\
\hline 44 & $n$ & $\begin{array}{r}4 \mathrm{I} \mathrm{y} \\
6 \mathrm{~m} \\
\end{array}$ & $\begin{array}{l}\mathbf{R} \\
\mathrm{L}\end{array}$ & $\begin{array}{l}I(+4) \\
I(H)\end{array}$ & 0.73 \\
\hline 45 & $"$ & $\begin{array}{l}39 \mathrm{y} \\
18 \mathrm{~m} \\
\end{array}$ & $\begin{array}{l}\mathbf{R} \\
\mathbf{L} \\
\end{array}$ & $\mid \begin{array}{l}2(H) \\
5(H)\end{array}$ & 0.98 \\
\hline 46 & $n$ & $\begin{array}{l}40 \mathrm{y} \\
10 \mathrm{~m}\end{array}$ & $\begin{array}{l}\mathbf{R} \\
\mathbf{L}\end{array}$ & $\left|\begin{array}{l}15(一) \\
16(-)\end{array}\right|$ & 1.34 \\
\hline
\end{tabular}




\begin{tabular}{|c|c|c|c|c|c|}
\hline 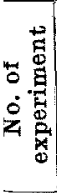 & 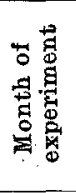 & 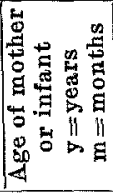 & 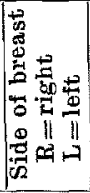 & 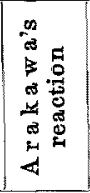 & 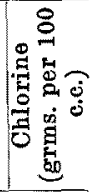 \\
\hline 47 & $\pi$ & $\begin{array}{r}29 \mathrm{~g} \\
4 \mathrm{~m}\end{array}$ & $\begin{array}{l}\mathrm{R} \\
\mathrm{L} \\
\end{array}$ & {$\left[\begin{array}{ll}11 & ( \pm) \\
11 & ( \pm)\end{array}\right.$} & 0.80 \\
\hline 48 & $n$ & $\begin{array}{l}28 \mathrm{y} \\
1 \mathrm{Im}\end{array}$ & $\begin{array}{l}\mathbf{R} \\
\mathbf{L}\end{array}$ & $\begin{array}{l}2(+) \\
2(+)\end{array}$ & 0.51 \\
\hline 49 & $"$ & $\begin{array}{r}21 \mathrm{y} \\
5 \mathrm{~m}\end{array}$ & $\begin{array}{l}\mathbf{R} \\
\mathrm{L}\end{array}$ & $\begin{array}{l}3( \pm) \\
8( \pm)\end{array}$ & 0.85 \\
\hline 50 & $"$ & $\begin{array}{l}29 \mathrm{y} \\
18 \mathrm{~m}\end{array}$ & $\begin{array}{l}\text { R } \\
\mathrm{L}\end{array}$ & $\begin{array}{l}3( \pm) \\
5(4)\end{array}$ & 0.87 \\
\hline 51 & $"$ & $\begin{array}{l}40 \mathrm{~g} \\
10 \mathrm{~m}\end{array}$ & $\begin{array}{l}\mathrm{R} \\
\mathrm{L}\end{array}$ & $\begin{array}{l}10( \pm) \\
16(H)\end{array}$ & 1.26 \\
\hline 52 & $"$ & $\begin{array}{r}94 \mathrm{y} \\
2 \mathrm{~m}\end{array}$ & $\begin{array}{l}\mathrm{R} \\
\mathrm{L}\end{array}$ & $\begin{array}{l}3( \pm) \\
5(+1)\end{array}$ & 0.92 \\
\hline 53 & $\Rightarrow$ & $\begin{array}{l}28 \mathrm{y} \\
16 \mathrm{~m}\end{array}$ & $\begin{array}{l}\mathrm{R} \\
\mathrm{L}\end{array}$ & $\begin{array}{l}11(-) \\
11(-)\end{array}$ & 0.60 \\
\hline 54 & $n$ & $\begin{array}{r}25 \mathrm{y} \\
5 \mathrm{~m}\end{array}$ & $\begin{array}{l}\mathbf{R} \\
\mathbf{L}\end{array}$ & $\mid \begin{array}{l}11( \pm) \\
11( \pm)\end{array}$ & 0.64 \\
\hline 55 & 7 & $\begin{array}{r}26 y \\
2 \mathrm{~m}\end{array}$ & $\begin{array}{l}\mathrm{R} \\
\mathrm{L}\end{array}$ & $\begin{array}{l}16(-) \\
16(-)\end{array}$ & 0.92 \\
\hline 56 & $n$ & $\begin{array}{r}25 \mathrm{y} \\
2 \mathrm{~m}\end{array}$ & $\begin{array}{l}\mathbf{R} \\
\mathrm{L}\end{array}$ & $\begin{array}{l}8(-) \\
8(-)\end{array}$ & 0.81 \\
\hline 57 & $n$ & $\begin{array}{r}26 y \\
8 \mathrm{~m}\end{array}$ & $\begin{array}{l}\mathrm{R} \\
\mathbf{L}\end{array}$ & $\begin{array}{l}14(-) \\
13( \pm)\end{array}$ & 0.81 \\
\hline 58 & 》 & $\begin{array}{r}26 \mathrm{y} \\
8 \mathrm{~m}\end{array}$ & $\begin{array}{l}\mathrm{R} \\
\mathrm{L}\end{array}$ & $\begin{array}{l}16(-) \\
16(-)\end{array}$ & 0.75 \\
\hline 59 & $"$ & $\begin{array}{l}29 \mathrm{y} \\
13 \mathrm{~m}\end{array}$ & $\begin{array}{l}\mathrm{R} \\
\mathrm{L}\end{array}$ & $\begin{array}{r}10(-) \\
6(-)\end{array}$ & 0.89 \\
\hline 60 & $n$ & $\begin{array}{r}32 \mathrm{y} \\
4 \mathrm{~m}\end{array}$ & $\begin{array}{l}\cdot \mathbf{R} \\
\mathbf{L} \\
\end{array}$ & $\begin{array}{r}16(-) \\
5( \pm)\end{array}$ & 0.89 \\
\hline 61 & $n$ & $\begin{array}{l}25 \mathrm{y} \\
10 \mathrm{~m}\end{array}$ & $\begin{array}{l}\bar{R} \\
\mathrm{~L}\end{array}$ & $\mid \begin{array}{r}16(-) \\
5( \pm)\end{array}$ & 0.75 \\
\hline 62 & $n$ & $\begin{array}{l}21 \mathrm{y} \\
11 \mathrm{~m}\end{array}$ & $\begin{array}{l}\mathrm{R} \\
\mathrm{L}\end{array}$ & $\begin{array}{l}16(-) \\
14(-)\end{array}$ & 1.21 \\
\hline 63 & $n$ & $\begin{array}{r}25 \mathrm{~g} \\
1 \mathrm{~m} \\
\end{array}$ & $\begin{array}{l}\mathrm{R} \\
\mathrm{L} \\
\end{array}$ & $\begin{array}{r}16(-) \\
4(+)\end{array}$ & 0.53 \\
\hline 64 & $n$ & $\begin{array}{r}20 y \\
2 \mathrm{~m} \\
\end{array}$ & $\begin{array}{l}\overline{\mathbf{R}} \\
\mathrm{L}\end{array}$ & $\begin{array}{l}14(-) \\
15(-)\end{array}$ & 0.91 \\
\hline 65 & $n$ & $\begin{array}{l}28 \mathrm{~g} \\
14 \mathrm{~m}\end{array}$ & $\begin{array}{l}\mathrm{R} \\
\mathrm{L}\end{array}$ & $\begin{array}{r}15(-) \\
4(-)\end{array}$ & 0.91 \\
\hline 66 & $n$ & $\begin{array}{r}25 \mathrm{~g} \\
7 \mathrm{~m} \\
\end{array}$ & $\begin{array}{l}\mathbf{R} \\
\mathbf{L}\end{array}$ & $\begin{array}{l}16(-) \\
14(-)\end{array}$ & 0.53 \\
\hline 67 & $\pi$ & $\begin{array}{r}26 \mathrm{y} \\
9 \mathrm{~m} \\
\end{array}$ & $\begin{array}{l}\mathbf{R} \\
\mathbf{L}\end{array}$ & $\begin{array}{r}5( \pm) \\
14(-)\end{array}$ & 0.85 \\
\hline 68 & $n$ & $\begin{array}{r}25 \mathrm{~g} \\
3 \mathrm{~m} \\
\end{array}$ & $\begin{array}{l}R \\
\mathrm{~L} \\
\end{array}$ & $\begin{array}{l}16(-) \\
16(-)\end{array}$ & 0.85 \\
\hline 69 & $n$ & $\begin{array}{r}23 \mathrm{y} \\
3 \mathrm{~m}\end{array}$ & $\begin{array}{l}\mathrm{R} \\
\mathrm{L}\end{array}$ & $\begin{array}{l}4( \pm) \\
3(-)\end{array}$ & 1.14 \\
\hline 70 & $n$ & $\begin{array}{l}25 \mathrm{y} \\
18 \mathrm{~m}\end{array}$ & $\begin{array}{l}\mathbf{R} \\
\mathbf{L}\end{array}$ & $\left\{\begin{array}{l}16(-) \\
16(-)\end{array}\right.$ & 0.50 \\
\hline 71 & $\pi$ & $\begin{array}{r}23 \mathrm{~g} \\
9 \mathrm{~m}\end{array}$ & $\begin{array}{l}\mathrm{R} \\
\mathrm{L}\end{array}$ & $\begin{array}{l}1(-) \\
7(+)\end{array}$ & 1.04 \\
\hline
\end{tabular}

\begin{tabular}{|c|c|c|c|c|c|}
\hline 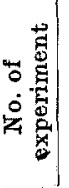 & 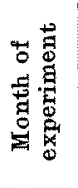 & 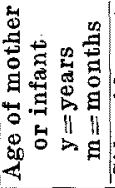 & 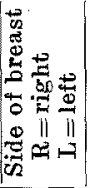 & 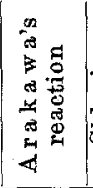 & 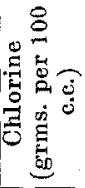 \\
\hline 72 & $n$ & $\begin{array}{l}28 \mathrm{y} \\
10 \mathrm{~m}\end{array}$ & $\begin{array}{l}\mathrm{R} \\
\mathrm{L}\end{array}$ & $\begin{array}{l}16(-) \\
16(-)\end{array}$ & 0.89 \\
\hline 73 & $\pi$ & $\begin{array}{c}25 \mathrm{y} \\
2 \mathrm{~m}\end{array}$ & $\begin{array}{l}\mathbf{R} \\
\mathrm{L}\end{array}$ & $\left|\begin{array}{l}12(-) \\
12(-)\end{array}\right|$ & 0.64 \\
\hline 74 & $n$ & $\begin{array}{r}28 \overline{\mathrm{y}} \\
1 \mathrm{~m}\end{array}$ & $\begin{array}{l}\mathbf{R} \\
\mathbf{L}\end{array}$ & $\left|\begin{array}{l}16(-) \\
15(-)\end{array}\right|$ & 0.43 \\
\hline 75 & $n$ & $\begin{array}{c}30 \mathrm{y} \\
6 \\
\end{array}$ & $\begin{array}{l}\mathrm{R} \\
\mathrm{L}\end{array}$ & {$\left[\begin{array}{l}14(-) \\
14(-)\end{array}\right]$} & 0.82 \\
\hline 76 & $n$ & $\begin{array}{r}25 \mathrm{y} \\
1 \mathrm{~m} \\
\end{array}$ & $\begin{array}{l}\mathbf{R} \\
\mathrm{L}\end{array}$ & $16(-)$ & 0.78 \\
\hline 77 & $n$ & $\begin{array}{r}32 \mathrm{~J} \\
6 \mathrm{~m} \\
\end{array}$ & $\begin{array}{l}\mathrm{R} \\
\mathrm{L}\end{array}$ & $\left|\begin{array}{r}10(+) \\
6(-)\end{array}\right|$ & 0.82 \\
\hline 78 & $n$ & $\begin{array}{r}24 \mathrm{~J} \\
5 \mathrm{~m} \\
\end{array}$ & $\begin{array}{l}\mathrm{R} \\
\mathrm{L}\end{array}$ & \begin{tabular}{|r|}
$3( \pm)$ \\
$4( \pm)$
\end{tabular} & 0.99 \\
\hline 79 & XII & $\begin{array}{r}23 \mathrm{y} \\
5 \mathrm{~m}\end{array}$ & $\begin{array}{l}\mathrm{R} \\
\mathrm{L}\end{array}$ & 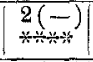 & 0.93 \\
\hline 80 & $n$ & $\begin{array}{l}40 \mathrm{y} \\
17 \mathrm{~m}\end{array}$ & $\begin{array}{l}\bar{R} \\
\mathrm{~L}\end{array}$ & $\begin{array}{l}7( \pm) \\
6(-)\end{array}$ & 0.59 \\
\hline 81 & $\pi$ & $\begin{array}{l}45 \mathrm{y} \\
20 \mathrm{~m}\end{array}$ & $\begin{array}{l}\bar{R} \\
\mathrm{~L}\end{array}$ & $\left|\begin{array}{l}16(-) \\
14(-)\end{array}\right|$ & 0.71 \\
\hline 82 & $n$ & $\begin{array}{r}29 \mathrm{y} \\
5 \mathrm{In}\end{array}$ & $\begin{array}{l}\mathrm{R} \\
\mathrm{L}\end{array}$ & $\begin{array}{l}7(+) \\
7(+)\end{array}$ & 0.76 \\
\hline 83 & $n$ & $\begin{array}{r}34 \mathrm{y} \\
2 \mathrm{~m} \\
\end{array}$ & $\begin{array}{l}\mathrm{R} \\
\mathrm{L} \\
\end{array}$ & $\begin{array}{l}14(-) \\
15(-)\end{array}$ & 0.39 \\
\hline 84 & » & $\begin{array}{r}25 \mathrm{y} \\
4 \mathrm{~m} \\
\end{array}$ & $\begin{array}{l}\mathrm{R} \\
\mathrm{L}\end{array}$ & $\begin{array}{l}16(-) \\
16(-)\end{array}$ & 0.44 \\
\hline 85 & $\pi$ & $\begin{array}{r}27 \mathrm{y} \\
4 \mathrm{~m} \\
\end{array}$ & $\begin{array}{l}\overline{\mathbf{R}} \\
\mathrm{L} \\
\end{array}$ & {$\left[\begin{array}{l}15(-) \\
15(-)\end{array}\right]$} & 0.50 \\
\hline 86 & $\pi$ & $\begin{array}{r}21 \mathrm{y} \\
2 \mathrm{~m} \\
\end{array}$ & $\begin{array}{l}\bar{R} \\
\mathrm{~L} \\
\end{array}$ & $\left|\begin{array}{l}15(-) \\
16(-)\end{array}\right|$ & 0.57 \\
\hline 87 & $n$ & $\begin{array}{l}27 \mathrm{y} \\
11 \mathrm{~m}\end{array}$ & $\begin{array}{l}\mathbf{R} \\
\mathbf{L}\end{array}$ & $\begin{array}{l}16(-) \\
16(-)\end{array}$ & 0.41 \\
\hline 88 & $\pi$ & $\begin{array}{l}24 \mathrm{y} \\
11 \mathrm{~m} \\
\end{array}$ & $\begin{array}{l}\mathbf{R} \\
\mathbf{L}\end{array}$ & $\left|\begin{array}{l}14(-) \\
10(+)\end{array}\right|$ & 0.82 \\
\hline 89 & $\eta$ & $\begin{array}{l}27 \mathrm{~g} \\
10 \mathrm{~m} \\
\end{array}$ & $\begin{array}{l}\mathbf{R} \\
\mathbf{L}\end{array}$ & $\left|\begin{array}{l}16(-) \\
16(-)\end{array}\right|$ & 0.89 \\
\hline 90 & 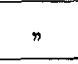 & $\begin{array}{l}41 \mathrm{y} \\
15 \mathrm{~m} \\
\end{array}$ & $\begin{array}{l}\mathbf{R} \\
\mathbf{L} \\
\end{array}$ & $\left|\begin{array}{l}15(-) \\
16(-)\end{array}\right|$ & 0.94 \\
\hline 91 & $\pi$ & $\begin{array}{l}29 \mathrm{y} \\
16 \mathrm{~m}\end{array}$ & $\begin{array}{l}\mathbf{R} \\
\mathbf{L}\end{array}$ & $\begin{array}{l}I(H) \\
I(H)\end{array}$ & 0.99 \\
\hline 92 & $n$ & $\begin{array}{l}41 \mathrm{~m} \\
19 \mathrm{~m}\end{array}$ & $\begin{array}{l}\mathbf{R} \\
\mathbf{L} \\
\end{array}$ & $\begin{array}{l}I(+) \\
1( \pm)\end{array}$ & 0.76 \\
\hline 93 & $\pi$ & $\begin{array}{l}34 \mathrm{~g} \\
12 \mathrm{~m} \\
\end{array}$ & $\begin{array}{l}\mathbf{R} \\
\mathbf{L}\end{array}$ & $\begin{array}{l}7( \pm) \\
8(-)\end{array}$ & 0.91 \\
\hline 94 & $\pi$ & $\begin{array}{r}20 \mathrm{y} \\
8 \mathrm{~m} \\
\end{array}$ & $\begin{array}{l}\mathrm{R} \\
\mathrm{L} \\
\end{array}$ & $\begin{array}{l}11( \pm) \\
11( \pm)\end{array}$ & 0.64 \\
\hline 95 & $n$ & $\begin{array}{l}26 \mathrm{y} \\
18 \mathrm{~m}\end{array}$ & $\begin{array}{l}\mathrm{IR} \\
\mathrm{I}\end{array}$ & $\begin{array}{l}6(-) \\
6( \pm)\end{array}$ & 1.02 \\
\hline 96 & $\pi$ & $\begin{array}{c}26 \mathrm{~g} \\
1 \mathrm{~m}\end{array}$ & $\begin{array}{l}\mathrm{R} \\
\mathrm{L}\end{array}$ & $\left|\begin{array}{l}12(-) \\
16(-)\end{array}\right|$ & 0.89 \\
\hline
\end{tabular}




\begin{tabular}{|c|c|c|c|c|c|}
\hline 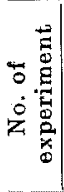 & 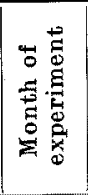 & 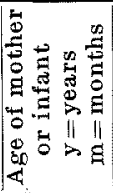 & 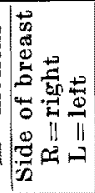 & 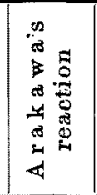 & 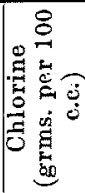 \\
\hline 97 & " & $\begin{array}{r}25 \mathrm{y} \\
2 \mathrm{~m} \\
\end{array}$ & $\begin{array}{l}\mathbf{R} \\
\mathrm{L}\end{array}$ & $10\left(\frac{-}{\xi}\right)$ & 0.82 \\
\hline 98 & $n$ & $\begin{array}{r}26 \mathrm{y} \\
2 \mathrm{~m}\end{array}$ & $\begin{array}{l}\mathrm{R} \\
\mathrm{L}\end{array}$ & $\begin{array}{r}15(-) \\
4(-)\end{array}$ & 1.01 \\
\hline 99 & $\pi$ & $\begin{array}{r}28 \mathrm{y} \\
4 \mathrm{~m}\end{array}$ & $\begin{array}{l}\mathbf{R} \\
\mathbf{L}\end{array}$ & $\begin{array}{l}16(-) \\
16(-)\end{array}$ & 0.80 \\
\hline 100 & $n$ & $\begin{array}{l}24 \mathrm{y} \\
19 \mathrm{~m} \\
\end{array}$ & $\begin{array}{l}\mathrm{R} \\
\mathrm{L}\end{array}$ & $\begin{array}{l}16(-) \\
16(-)\end{array}$ & 0.99 \\
\hline 101 & $y$ & $\begin{array}{l}27 \mathrm{y} \\
21 \mathrm{~m} \\
\end{array}$ & $\begin{array}{l}\mathbf{R} \\
\mathbf{L}\end{array}$ & $\begin{array}{l}5(+1) \\
4(+) \\
\end{array}$ & 0.82 \\
\hline 102 & " & $\begin{array}{l}24 \mathrm{~g} \\
10 \mathrm{~m}\end{array}$ & $\begin{array}{l}\mathbf{R} \\
\mathbf{L}\end{array}$ & $\begin{array}{l}16(-) \\
16(-)\end{array}$ & 0.57 \\
\hline 103 & $n$ & $\begin{array}{l}23 \mathrm{y} \\
14 \mathrm{~m} \\
\end{array}$ & $\begin{array}{l}\mathbf{R} \\
\mathrm{L} \\
\end{array}$ & $\begin{array}{l}16(-) \\
14(-)\end{array}$ & 1.08 \\
\hline 104 & » & $\begin{array}{r}26 \mathrm{y} \\
1 \mathrm{~m} \\
\end{array}$ & $\begin{array}{l}\mathrm{R} \\
\mathrm{L}\end{array}$ & {$\left[\begin{array}{l}14(-) \\
16(-)\end{array}\right.$} & 0.75 \\
\hline 105 & $\pi$ & $\begin{array}{l}31 \mathrm{y} \\
10 \mathrm{~m}\end{array}$ & $\begin{array}{l}\mathbf{R} \\
\mathbf{L} \\
\end{array}$ & $\begin{array}{l}4(-) \\
4(-)\end{array}$ & 0.50 \\
\hline 106 & $"$ & $\begin{array}{r}23 \mathrm{y} \\
2 \mathrm{~m} \\
\end{array}$ & $\begin{array}{l}\mathbf{R} \\
\mathbf{L}\end{array}$ & $\left|\begin{array}{r}11(+) \\
7(-)\end{array}\right|$ & 0.80 \\
\hline 107 & " & $\begin{array}{l}28 \mathrm{~g} \\
15 \mathrm{~m}\end{array}$ & $\begin{array}{l}\mathrm{R} \\
\mathrm{L}\end{array}$ & $\left|\begin{array}{l}16(-) \\
16(--)\end{array}\right|$ & 1.16 \\
\hline 108 & $\pi$ & $\begin{array}{r}26 \mathrm{y} \\
2 \mathrm{~m} \\
\end{array}$ & $\begin{array}{l}\mathrm{R} \\
\mathrm{L} \\
\end{array}$ & $\begin{array}{l}7(-) \\
7(-)\end{array}$ & 1.14 \\
\hline 109 & $n$ & $\begin{array}{r}30 \mathrm{y} \\
2 \mathrm{~m}\end{array}$ & $\begin{array}{l}\mathbf{R} \\
\mathrm{L}\end{array}$ & $\left|\begin{array}{l}16(-) \\
16(-)\end{array}\right|$ & 0.72 \\
\hline 110 & $n$ & $\begin{array}{r}31 \mathrm{y} \\
7 \mathrm{~m} \\
\end{array}$ & $\begin{array}{l}\mathrm{K} \\
\mathrm{L}\end{array}$ & $\mid \begin{array}{l}8(-) \\
7( \pm)\end{array}$ & 0.71 \\
\hline 111 & $\begin{array}{c}1 \\
(1937) \\
\end{array}$ & $\begin{array}{c}34 \mathrm{y} \\
1 \mathrm{~m} \\
\end{array}$ & $\begin{array}{l}\bar{R} \\
\mathrm{~L} \\
\end{array}$ & $\left|\begin{array}{l}12(-) \\
12(-)\end{array}\right|$ & 1.17 \\
\hline 112 & $\pi$ & $\begin{array}{r}31 \mathrm{y} \\
7 \mathrm{~m} \\
\end{array}$ & $\begin{array}{l}\mathrm{R} \\
\mathrm{I}\end{array}$ & $\left|\begin{array}{l}14(-) \\
16(-)\end{array}\right|$ & 0.86 \\
\hline 113 & $n$ & $\begin{array}{l}27 \mathrm{y} \\
14 \mathrm{~m}\end{array}$ & $\begin{array}{l}\mathrm{R} \\
\mathrm{L}\end{array}$ & $\left|\begin{array}{l}6( \pm) \\
6( \pm)\end{array}\right|$ & 0.85 \\
\hline 114 & $\pi$ & $\begin{array}{r}23 \mathrm{~g} \\
1 \mathrm{~m} \\
\end{array}$ & $\begin{array}{l}\mathbf{R} \\
\mathrm{L} \\
\end{array}$ & $\begin{array}{l}15(-) \\
14(-)\end{array}$ & 0.64 \\
\hline 115 & $n$ & $\begin{array}{r}32 y \\
3 \mathrm{~m} \\
\end{array}$ & $\begin{array}{l}\mathrm{R} \\
\mathrm{L} \\
\end{array}$ & $\begin{array}{l}16(-) \\
16(-) \\
\end{array}$ & 0.97 \\
\hline 116 & $n$ & $\begin{array}{r}26 \mathrm{y} \\
2 \mathrm{~m} \\
\end{array}$ & $\begin{array}{l}\mathrm{R} \\
\mathrm{L} \\
\end{array}$ & $\begin{array}{r}\$ \$ \\
\$ \$ 8 \\
\end{array}$ & 0.53 \\
\hline 117 & $\pi$ & $\begin{array}{r}25 \mathrm{~g} \\
5 \mathrm{~m} \\
\end{array}$ & $\begin{array}{l}\mathbf{R} \\
\mathrm{L} \\
\end{array}$ & $\begin{array}{l}15(-) \\
13(-)\end{array}$ & 0.89 \\
\hline 118 & $\therefore$ & $\begin{array}{r}31 \mathrm{y} \\
3 \mathrm{~m} \\
\end{array}$ & $\begin{array}{l}\mathbf{R} \\
\mathrm{L} \\
\end{array}$ & $\begin{array}{l}12(-) \\
12(-)\end{array}$ & 0.89 \\
\hline 119 & $n$ & $\begin{array}{r}29 \mathrm{y} \\
4 \mathrm{~m} \\
\end{array}$ & $\begin{array}{l}\mathrm{R} \\
\mathrm{L}\end{array}$ & $\begin{array}{l}6( \pm) \\
6( \pm)\end{array}$ & 1.08 \\
\hline 120 & * & $\begin{array}{r}25 \mathrm{y} \\
7 \mathrm{~m} \\
\end{array}$ & $\begin{array}{l}\mathrm{K} \\
\mathrm{L}\end{array}$ & $\begin{array}{l}16(-) \\
16(-)\end{array}$ & 0.78 \\
\hline 121 & $n$ & $\begin{array}{c}23 \mathrm{y} \\
9 \mathrm{~m}\end{array}$ & $\begin{array}{l}\mathrm{R} \\
\mathrm{L}\end{array}$ & $\begin{array}{l}2(+) \\
5(+)\end{array}$ & 0.83 \\
\hline
\end{tabular}

\begin{tabular}{|c|c|c|c|c|c|}
\hline 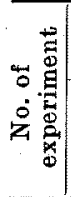 & 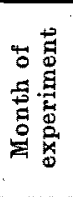 & 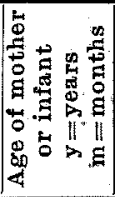 & 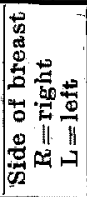 & 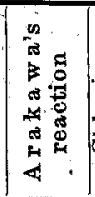 & 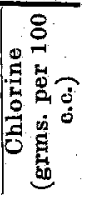 \\
\hline 122 & $n$ & $\begin{array}{r}27 \mathrm{~g} \\
2 \mathrm{~m}\end{array}$ & $\begin{array}{l}\mathbf{R} \\
\mathrm{L}\end{array}$ & $\begin{array}{l}6(-) \\
6(-)\end{array}$ & 1.10 \\
\hline 123 & $n$ & $\begin{array}{l}29 \mathrm{y} \\
16 \mathrm{~m}\end{array}$ & $\begin{array}{l}\mathrm{R} \\
\mathrm{L}\end{array}$ & \begin{tabular}{|l|}
$16(-)$ \\
$16(-)$
\end{tabular} & 0.78 \\
\hline 124 & " & $\begin{array}{l}25 \mathrm{y} \\
15 \mathrm{~m}\end{array}$ & $\begin{array}{l}\mathrm{R} \\
\mathrm{L}\end{array}$ & $\left|\begin{array}{l}16(-) \\
16(-)\end{array}\right|$ & 0.24 \\
\hline 125 & $\pi$ & $\begin{array}{l}30 \mathrm{y} \\
15 \mathrm{~m}\end{array}$ & $\begin{array}{l}\mathrm{R} \\
\mathrm{L}\end{array}$ & $\begin{array}{l}16(-) \\
16(-)\end{array}$ & 0.93 \\
\hline 126 & $\pi$ & $\begin{array}{l}25 \mathrm{y} \\
10 \mathrm{~m}\end{array}$ & $\begin{array}{l}\mathrm{R} \\
\mathrm{L}\end{array}$ & $\left|\begin{array}{r}6(-) \\
12(-)\end{array}\right|$ & 1.07 \\
\hline 127 & $n$ & $\begin{array}{r}25 \mathrm{y} \\
5 \mathrm{~m}\end{array}$ & $\begin{array}{l}\mathbf{R} \\
\mathrm{L}\end{array}$ & $\left|\begin{array}{l}16(-) \\
16(-)\end{array}\right|$ & 0.71 \\
\hline 128 & $n$ & $\begin{array}{r}31 \mathrm{y} \\
3 \mathrm{~m} \\
\end{array}$ & $\begin{array}{l}\mathbf{R} \\
\mathbf{L}\end{array}$ & $\left|\begin{array}{l}11( \pm) \\
11( \pm)\end{array}\right|$ & 0.89 \\
\hline 129 & $\pi$ & $\begin{array}{l}30 \mathrm{y} \\
15 \mathrm{~m} \\
\end{array}$ & $\begin{array}{l}\mathbf{R} \\
\mathrm{L} \\
\end{array}$ & $\begin{array}{l}16(-) \\
11( \pm)\end{array}$ & 0,75 \\
\hline 130 & $n$ & $\begin{array}{r}28 \mathrm{y} \\
3 \mathrm{~m} \\
\end{array}$ & $\begin{array}{l}\mathbf{R} \\
\mathbf{L}\end{array}$ & $\begin{array}{r}16(-) \\
6(+)\end{array}$ & 1.03 \\
\hline 131 & $n$ & $\begin{array}{r}27 \mathrm{y} \\
2 \mathrm{~m}\end{array}$ & $\begin{array}{l}\mathrm{R} \\
\mathrm{L}\end{array}$ & $\left|\begin{array}{l}16(-) \\
16(-)\end{array}\right|$ & 0.58 \\
\hline 132 & $n$ & $\begin{array}{l}32 \mathrm{y} \\
15 \mathrm{~m}\end{array}$ & $\begin{array}{l}\mathrm{R} \\
\mathrm{L}\end{array}$ & $\begin{array}{l}12(-) \\
16(-)\end{array} \mid$ & 0.82 \\
\hline 133 & $\pi$ & $\begin{array}{l}26 \mathrm{y} \\
12 \mathrm{~m}\end{array}$ & $\begin{array}{l}\mathrm{R} \\
\mathrm{L}\end{array}$ & $\left|\begin{array}{l}16(-) \\
10( \pm)\end{array}\right|$ & 0.71 \\
\hline 134 & $n$ & $\begin{array}{l}25 \mathrm{y} \\
13 \mathrm{~m}\end{array}$ & $\begin{array}{l}\mathbf{R} \\
\mathrm{I}\end{array}$ & $\begin{array}{r}16(-) \\
8(-)\end{array}$ & 0.68 \\
\hline 135 & $"$ & $\begin{array}{r}25 \mathrm{y} \\
3 \mathrm{~m} \\
\end{array}$ & $\begin{array}{l}\mathrm{R} \\
\mathrm{L}\end{array}$ & \begin{tabular}{|l|}
$11( \pm)$ \\
$11( \pm)$
\end{tabular} & 0.99 \\
\hline 136 & $"$ & $\begin{array}{l}25 \mathrm{y} \\
10 \mathrm{~m}\end{array}$ & $\begin{array}{l}\mathrm{R} \\
\mathrm{L}\end{array}$ & $\mid \begin{array}{r}3( \pm) \\
16( \pm)\end{array}$ & 0.92 \\
\hline 137 & $\eta$ & $\begin{array}{r}30 \mathrm{y} \\
7 \mathrm{~m} \\
\end{array}$ & $\begin{array}{l}\mathrm{R} \\
\mathrm{L}\end{array}$ & $\begin{array}{r}9(+) \\
14( \pm)\end{array}$ & 0.82 \\
\hline 138 & 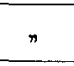 & $\begin{array}{r}33 \mathrm{y} \\
7 \mathrm{~m}\end{array}$ & $\begin{array}{l}\mathbf{R} \\
\mathrm{L} \\
\end{array}$ & \begin{tabular}{|l|}
$16(-)$ \\
$16(-)$
\end{tabular} & 0.21 \\
\hline 139 & $n$ & $\begin{array}{l}34 \mathrm{y} \\
12 \mathrm{~m} \\
\end{array}$ & $\begin{array}{l}\mathrm{R} \\
\mathrm{L}\end{array}$ & $\begin{array}{l}16(-) \\
12(-)\end{array}$ & 0.96 \\
\hline 140 & $n$ & $\begin{array}{l}27 \mathrm{y} \\
10 \mathrm{~m}\end{array}$ & $\begin{array}{l}\mathrm{R} \\
\mathrm{L}\end{array}$ & $\begin{array}{l}16(-) \\
15(-)\end{array}$ & 0.11 \\
\hline 141 & $n$ & $\begin{array}{r}26 \mathrm{y} \\
9 \mathrm{~m}\end{array}$ & $\begin{array}{l}\mathbf{R} \\
\mathrm{L}\end{array}$ & \begin{tabular}{|l|}
$16(-)$ \\
$16(-)$
\end{tabular} \mid & 0.76 \\
\hline 142 & $\pi$ & $\begin{array}{r}28 \mathrm{y} \\
4 \mathrm{~m} \\
\end{array}$ & $\begin{array}{l}\mathbf{R} \\
\mathbf{L}\end{array}$ & $\begin{array}{l}16(-) \\
16(-)\end{array}$ & 0.10 \\
\hline 143 & $n$ & $\begin{array}{r}27 \mathrm{y} \\
9 \mathrm{~m}\end{array}$ & $\begin{array}{l}\mathrm{R} \\
\mathrm{L}\end{array}$ & $\left|\begin{array}{l}16(-) \\
16(-)\end{array}\right|$ & 0.11 \\
\hline 144 & $n$ & $\begin{array}{r}29 \mathrm{y} \\
6 \mathrm{~m} \\
\end{array}$ & $\begin{array}{l}\mathbf{R} \\
\mathbf{L} \\
\end{array}$ & $\left|\begin{array}{l}15(-) \\
16(-)\end{array}\right|$ & 0.92 \\
\hline 145 & $\pi$ & $\begin{array}{l}28 \mathrm{y} \\
12 \mathrm{~m}\end{array}$ & $\begin{array}{l}\overline{\mathbf{K}} \\
\mathrm{L}\end{array}$ & $\left|\begin{array}{l}4(-) \\
8(-)\end{array}\right|$ & 0.68 \\
\hline 146 & $"$ & $\begin{array}{l}27 \mathrm{~g} \\
11 \mathrm{~m}\end{array}$ & $\begin{array}{l}\mathrm{R} \\
\mathrm{L}\end{array}$ & $\left|\begin{array}{r}10( \pm) \\
6(+)\end{array}\right|$ & 0.60 \\
\hline
\end{tabular}




\begin{tabular}{|c|c|c|c|c|c|}
\hline 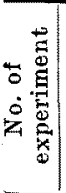 & 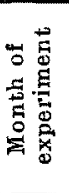 & 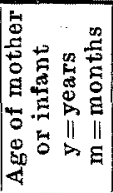 & 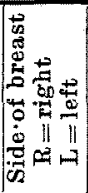 & 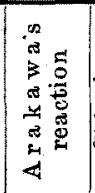 & 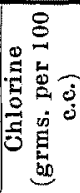 \\
\hline 147 & $\eta$ & $\begin{array}{r}22 \mathrm{y} \\
4 \mathrm{~m}\end{array}$ & $\begin{array}{l}\overline{\mathbf{R}} \\
\mathbf{L}\end{array}$ & $\left|\begin{array}{r}12(-) \\
3(-)\end{array}\right|$ & 0.94 \\
\hline 148 & $n$ & $\begin{array}{r}33 \mathrm{y} \\
6 \mathrm{~m}\end{array}$ & $\begin{array}{l}\mathbf{K} \\
\mathbf{L}\end{array}$ & $\left|\begin{array}{r}16(-) \\
\cdot 1(+)\end{array}\right|$ & 0.90 \\
\hline 149 & $\pi$ & $\begin{array}{r}25 \mathrm{y} \\
2 \mathrm{~m}\end{array}$ & $\begin{array}{l}\mathrm{K} \\
\mathrm{L}\end{array}$ & $\left|\begin{array}{c}8(-) \\
16(-)\end{array}\right|$ & 0.98 \\
\hline 150 & $\pi$ & $\begin{array}{r}25 \mathrm{y} \\
1 \mathrm{~m} \\
\end{array}$ & $\begin{array}{l}\mathbf{K} \\
\mathrm{L}\end{array}$ & $\left|\begin{array}{l}16(-) \\
16(-)\end{array}\right|$ & 0.84 \\
\hline 151 & $\pi$ & $\begin{array}{l}28 \mathrm{y} \\
11 \mathrm{~m}\end{array}$ & $\begin{array}{l}\mathbf{R} \\
\mathbf{L}\end{array}$ & $\left|\begin{array}{l}7( \pm) \\
7( \pm)\end{array}\right|$ & 0.84 \\
\hline 252 & $\pi$ & $\begin{array}{l}24 \mathrm{y} \\
10 \mathrm{~m}\end{array}$ & $\begin{array}{l}\mathbf{R} \\
\mathrm{L}\end{array}$ & $\left|\begin{array}{r}7( \pm) \\
11(-)\end{array}\right|$ & 1.19 \\
\hline 153 & 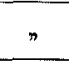 & $\begin{array}{r}25 \mathrm{y} \\
2 \mathrm{~m} \\
\end{array}$ & $\begin{array}{l}\mathrm{R} \\
\mathrm{L}\end{array}$ & $\begin{array}{r}6(-) \\
10(-)\end{array}$ & 0.85 \\
\hline 154 & $n$ & $\begin{array}{r}22 \mathrm{y} \\
7 \mathrm{~m}\end{array}$ & L & $\begin{array}{l}9(+) \\
4(-)\end{array}$ & 1.03 \\
\hline 155 & II & $\begin{array}{r}28 \mathrm{y} \\
3 \mathrm{~m}\end{array}$ & $\begin{array}{l}\mathrm{R} \\
\mathrm{L}\end{array}$ & $\left|\begin{array}{c}8 s 58 \\
13( \pm)\end{array}\right|$ & 1.04 \\
\hline 156 & n & $\begin{array}{r}25 \mathrm{y} \\
4 \mathrm{~m}\end{array}$ & $\begin{array}{l}\mathbf{R} \\
\mathbf{L}\end{array}$ & $\left|\begin{array}{l}16(-) \\
16(-)\end{array}\right|$ & 0.57 \\
\hline 157 & $n$ & $\begin{array}{r}25 \mathrm{y} \\
9 \mathrm{~m}\end{array}$ & $\begin{array}{l}\mathrm{R} \\
\mathrm{L}\end{array}$ & $\begin{array}{l}12(-) \\
16(-)\end{array}$ & 0.50 \\
\hline 158 & 7 & $\begin{array}{r}28 \mathrm{y} \\
6 \mathrm{~m} \\
\end{array}$ & $\begin{array}{l}\mathbf{R} \\
\mathbf{L}\end{array}$ & $\left|\begin{array}{r}16(-) \\
4(-)\end{array}\right|$ & 0.92 \\
\hline 159 & $n$ & $\begin{array}{r}34 \mathrm{y} \\
6 \mathrm{~m} \\
\end{array}$ & $\begin{array}{l}\mathrm{R} \\
\mathrm{L}\end{array}$ & $\begin{array}{l}2(\mathrm{H}) \\
4(+)\end{array}$ & 0.75 \\
\hline 160 & $n$ & $\begin{array}{l}34 \mathrm{~g} \\
17 \mathrm{~m} \\
\end{array}$ & $\begin{array}{l}\mathrm{P} \\
\mathrm{L}\end{array}$ & {$\left[\begin{array}{r}16(-) \\
8(-)\end{array}\right]$} & 0.69 \\
\hline 161 &.$m$ & $\begin{array}{r}28 \mathrm{y} \\
8 \mathrm{~m}\end{array}$ & $\begin{array}{l}\mathrm{R} \\
\mathbf{L}\end{array}$ & $\begin{array}{r}16(-) \\
6(-)\end{array}$ & 0.82 \\
\hline 62 & $\pi$ & $\begin{array}{r}36 \mathrm{y} \\
9 \mathrm{~m} \\
\end{array}$ & $\begin{array}{l}\mathbf{R} \\
\mathbf{L}\end{array}$ & $\begin{array}{l}6(-) \\
7( \pm)\end{array}$ & 1.21 \\
\hline 63 & \# & $\begin{array}{r}30 \mathrm{y} \\
4 \mathrm{~m} \\
\end{array}$ & $\begin{array}{l}\mathbf{R} \\
\mathrm{L}\end{array}$ & $\left|\begin{array}{l}16(-) \\
16(-)\end{array}\right|$ & 0.64 \\
\hline 84 & $n$ & $\begin{array}{l}25 \mathrm{y} \\
26 \mathrm{~m}\end{array}$ & $\begin{array}{l}\mathbf{R} \\
\mathrm{L}\end{array}$ & $\left|\begin{array}{l}16(-) \\
16(-)\end{array}\right|$ & 0.41 \\
\hline 55 & $n$ & $\begin{array}{r}34 \mathrm{y} \\
4 \mathrm{~m}\end{array}$ & $\begin{array}{l}\mathbf{R} \\
\mathrm{L}\end{array}$ & $\begin{array}{r}15( \pm) \\
7( \pm)\end{array}$ & 0.83 \\
\hline 36 & $\pi$ & $\begin{array}{c}23 \mathrm{y} \\
9 \mathrm{~m}\end{array}$ & $\begin{array}{l}\mathrm{R} \\
\mathrm{L}\end{array}$ & $\left|\begin{array}{r}10(+) \\
5(+t)\end{array}\right|$ & 0.89 \\
\hline 87 & $\pi$ & $\begin{array}{r}25 \mathrm{y} \\
9 \mathrm{~m} \\
\end{array}$ & $\begin{array}{l}\mathbf{R} \\
\mathbf{L}\end{array}$ & $12(-)$ & 0.89 \\
\hline 8 & $n$ & $\begin{array}{r}30 \mathrm{y} \\
1 \mathrm{~m} \\
\end{array}$ & $\begin{array}{l}\mathrm{R} \\
\mathrm{L}\end{array}$ & $\left|\begin{array}{c}15(-) \\
85888\end{array}\right|$ & 0.71 \\
\hline 59 & $\pi$ & $\begin{array}{r}29 \mathrm{y} \\
6 \mathrm{~m} \\
\end{array}$ & $\begin{array}{l}\mathbf{R} \\
\mathbf{L}\end{array}$ & $\begin{array}{r}11( \pm) \\
9(+)\end{array}$ & 0.89 \\
\hline 170 & $\Rightarrow$ & $\begin{array}{r}25 \mathrm{y} \\
5 \mathrm{~m} \\
\end{array}$ & $\begin{array}{l}\mathrm{R} \\
\mathrm{I}\end{array}$ & $\begin{array}{l}7( \pm) \\
5(t)\end{array}$ & 0.99 \\
\hline & $n$ & $\begin{array}{r}26 \mathrm{y} \\
9 \mathrm{~m}\end{array}$ & $\begin{array}{l}\bar{R} \\
\mathrm{~L}\end{array}$ & $\begin{array}{r}5(+) \\
10(+)\end{array}$ & 0.92 \\
\hline
\end{tabular}

\begin{tabular}{|c|c|c|c|c|c|}
\hline 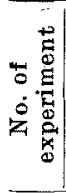 & 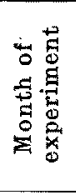 & 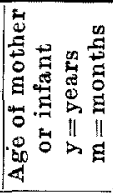 & 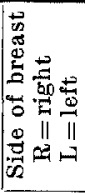 & 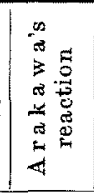 & 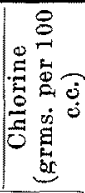 \\
\hline 172 & $\pi$ & $\begin{array}{r}30 \mathrm{y} \\
9 \mathrm{~m} \\
\end{array}$ & $\begin{array}{l}\mathrm{K} \\
\mathrm{L}\end{array}$ & $\begin{array}{l}4(+) \\
5( \pm)\end{array}$ & .96 \\
\hline 173 & " & $\begin{array}{l}29 \mathrm{y} \\
11 \mathrm{~m}\end{array}$ & $\begin{array}{l}\mathbf{K} \\
\mathbf{L}\end{array}$ & $\begin{array}{l}9(+) \\
4(+)\end{array}$ & .85 \\
\hline 174 & $n$ & $\begin{array}{r}23 \mathrm{y} \\
1 \mathrm{~m}\end{array}$ & $\begin{array}{l}\mathbf{R} \\
\mathbf{L}\end{array}$ & $\begin{array}{r}3(-) \\
15( \pm)\end{array}$ & 0.62 \\
\hline 75 & $n$ & $\begin{array}{r}25 \mathrm{y} \\
9 \mathrm{~m}\end{array}$ & $\begin{array}{l}\mathbf{R} \\
\mathbf{L}\end{array}$ & $\begin{array}{r}3(-) \\
14(-)\end{array}$ & 1.08 \\
\hline 176 & $\#$ & $\begin{array}{r}23 \mathrm{y} \\
6 \mathrm{~m}\end{array}$ & $\begin{array}{l}\mathbf{R} \\
\mathbf{L}\end{array}$ & $\begin{array}{l}1(+1) \\
6( \pm)\end{array}$ & 0.82 \\
\hline 177 & $\Rightarrow$ & $\begin{array}{l}23 \mathrm{y} \\
10 \mathrm{~m}\end{array}$ & $\begin{array}{l}\mathbf{R} \\
\mathbf{L} \\
\end{array}$ & $\mid \begin{array}{l}15( \pm) \\
11(+)\end{array}$ & 0.82 \\
\hline 178 & " & $\begin{array}{l}33 \mathrm{y} \\
12 \mathrm{~m}\end{array}$ & $\begin{array}{l}\mathrm{R} \\
\mathrm{L}\end{array}$ & $\left|\begin{array}{l}11(+) \\
15( \pm)\end{array}\right|$ & 0.78 \\
\hline 79 & \# & $\begin{array}{l}38 \mathrm{y} \\
20 \mathrm{~m}\end{array}$ & $\begin{array}{l}\mathrm{R} \\
\mathrm{L}\end{array}$ & $\begin{array}{l}7(+) \\
7(-)\end{array}$ & .0 .99 \\
\hline 30 & $\pi$ & $\begin{array}{l}35 \mathrm{y} \\
20 \mathrm{~m}\end{array}$ & $\begin{array}{l}\mathbf{R} \\
\mathbf{L}\end{array}$ & $\begin{array}{l}4(+) \\
4(+)\end{array}$ & 0.85 \\
\hline 81 & $"$ & $\begin{array}{r}26 \mathrm{y} \\
2 \mathrm{~m}\end{array}$ & $\begin{array}{l}\mathbf{R} \\
\mathbf{L}\end{array}$ & $\left|\begin{array}{r}5( \pm) \\
14( \pm)\end{array}\right|$ & 0.92 \\
\hline 82 & n & $\begin{array}{l}32 \mathrm{y} \\
15 \mathrm{~m}\end{array}$ & $\begin{array}{l}\mathbf{R} \\
\mathbf{L}\end{array}$ & $\begin{array}{l}4(+) \\
5(+)\end{array}$ & .17 \\
\hline 183 & " & $\begin{array}{r}27 \mathrm{y} \\
2 \mathrm{~m} \\
\end{array}$ & $\begin{array}{l}\mathbf{R} \\
\mathbf{L}\end{array}$ & $\begin{array}{l}4(+) \\
4(+)\end{array}$ & .96 \\
\hline 184 & $n$ & $\begin{array}{r}31 \mathrm{y} \\
5 \mathrm{~m} \\
\end{array}$ & $\begin{array}{l}\mathbf{R} \\
\mathbf{L} \\
\end{array}$ & $\begin{array}{l}7( \pm) \\
7( \pm)\end{array}$ & 1.17 \\
\hline 35 & $n$ & $\begin{array}{r}32 \mathrm{y} \\
4 \mathrm{~m} \\
\end{array}$ & $\begin{array}{l}\mathrm{R} \\
\mathbf{L}\end{array}$ & $\left|\begin{array}{r}8(-) \\
16(-)\end{array}\right|$ & 6 \\
\hline 186 & $\#$ & $\begin{array}{l}26 \mathrm{y} \\
22 \mathrm{~m}\end{array}$ & $\begin{array}{l}\mathbf{R} \\
\mathrm{L}\end{array}$ & $\begin{array}{l}14(-) \\
16(-)\end{array}$ & .7 \\
\hline 87 & $n$ & $\begin{array}{r}26 \mathrm{~g} \\
7 \mathrm{~m} \\
\end{array}$ & $\begin{array}{l}\mathbf{R} \\
\mathrm{L}\end{array}$ & $\mid \begin{array}{l}11(+) \\
11(+)\end{array}$ & 99 \\
\hline 88 & III & $\begin{array}{r}30 \mathrm{y} \\
6 \mathrm{~m} \\
\end{array}$ & $\begin{array}{l}\mathbf{R} \\
\mathbf{L}\end{array}$ & $\left|\begin{array}{l}10(-) \\
16(-)\end{array}\right|$ & .70 \\
\hline 39 & $n$ & $\begin{array}{r}24 \mathrm{y} \\
4 \mathrm{~m} \\
\end{array}$ & $\begin{array}{l}\mathrm{R} \\
\mathrm{L}\end{array}$ & $\begin{array}{l}7(+) \\
7(+)\end{array}$ & 81 \\
\hline 90 & $n$ & $\begin{array}{l}29 \mathrm{y} \\
19 \mathrm{~m}\end{array}$ & $\begin{array}{l}\mathbf{R} \\
\mathbf{L} \\
\end{array}$ & $\left|\begin{array}{l}12(-) \\
12(-)\end{array}\right|$ & .87 \\
\hline 31 & $\pi$ & $\begin{array}{r}27 \mathrm{y} \\
2 \mathrm{~m} \\
\end{array}$ & $\begin{array}{l}\mathrm{K} \\
\mathrm{L}\end{array}$ & $\left|\begin{array}{l}16(-) \\
16(-)\end{array}\right|$ & 0.36 \\
\hline 92 & $n$ & $\begin{array}{r}25 \mathrm{y} \\
3 \mathrm{~m}\end{array}$ & $\begin{array}{l}\mathrm{R} \\
\mathrm{L}\end{array}$ & $\begin{array}{l}4(+) \\
4(+)\end{array}$ & .00 \\
\hline 93 & $n$ & $\begin{array}{r}29 \mathrm{y} \\
5 \mathrm{~m} \\
\end{array}$ & $\begin{array}{l}\mathbf{R} \\
\mathrm{L}\end{array}$ & $\left|\begin{array}{r}11(+) \\
4(+)\end{array}\right|$ & 1.0 \\
\hline 94 & $n$ & $\begin{array}{c}23 \mathrm{y} \\
1 \mathrm{~m}\end{array}$ & $\begin{array}{l}\mathrm{K} \\
\mathbf{L}\end{array}$ & $\left|\begin{array}{r}7( \pm) \\
16(-)\end{array}\right|$ & 0.8 \\
\hline 95 & $n$ & $\begin{array}{r}27 \mathrm{y} \\
2 \mathrm{~m} \\
\end{array}$ & $\begin{array}{l}\mathrm{R} \\
\mathrm{L}\end{array}$ & $\left|\begin{array}{l}10(+) \\
10+)\end{array}\right|$ & 1.26 \\
\hline & & $\begin{array}{r}23 \mathrm{y} \\
3 \mathrm{~m}\end{array}$ & $\mathrm{k}$ & $\begin{array}{l}1(H) \\
1(H)\end{array}$ & 1.0 \\
\hline
\end{tabular}




\begin{tabular}{|c|c|c|c|c|c|}
\hline ए. & 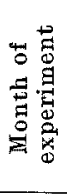 & 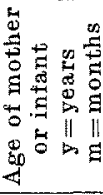 & 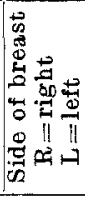 & 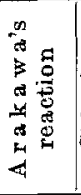 & 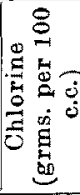 \\
\hline 97 & n & $\begin{array}{r}27 \mathrm{~g} \\
9 \mathrm{~m} \\
\end{array}$ & $\begin{array}{l}\mathrm{R} \\
\mathrm{L}\end{array}$ & $\begin{array}{l}1(H) \\
2(H)\end{array}$ & 1.17 \\
\hline 98 & " & $\begin{array}{r}25 \text { y } \\
7 \mathrm{~m}\end{array}$ & L & $\begin{array}{l}1(\#) \\
2(\#)\end{array}$ & 1.27 \\
\hline 199 & " & $\begin{array}{r}22 y \\
8 \mathrm{~m}\end{array}$ & $\begin{array}{l}\mathbf{R} \\
\mathrm{L}\end{array}$ & $\begin{array}{l}1(+t) \\
1(+t)\end{array}$ & 1.1 \\
\hline 00 & " & $\begin{array}{r}24 \mathrm{y} \\
8 \mathrm{~m}\end{array}$ & $\begin{array}{l}\mathrm{R} \\
\mathrm{L}\end{array}$ & $\begin{array}{l}1(H) \\
1(H)\end{array}$ & 1.34 \\
\hline 01 & $\pi$ & $\begin{array}{l}26 \mathrm{y} \\
12 \mathrm{~m}\end{array}$ & $\begin{array}{l}\mathrm{R} \\
\mathrm{L}\end{array}$ & $\begin{array}{l}1(H) \\
1(H)\end{array}$ & 1.1 \\
\hline & & $\begin{array}{r}25 y \\
8 \mathrm{~m}\end{array}$ & $\mathbf{R}$ & $\begin{array}{l}1(H) \\
2(H)\end{array}$ & 1,36 \\
\hline
\end{tabular}

* Cf. Table 4.

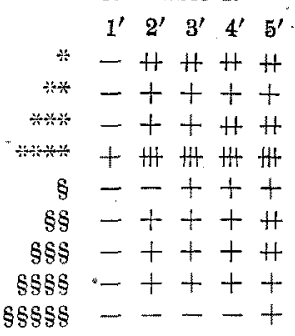

Table 4.

Table of signs for showing different A rak awa's reaction.

$1^{\prime} \quad 2^{\prime} \quad 3^{\prime} 4^{\prime} \quad 5^{\prime}$

1. $1(\mathrm{H})$ stands for $\mathrm{HH} \mathrm{H}+\mathrm{H}+\mathrm{H}$

2. $I(H) n$ H H H H H

3. $2(H)$ H H H H H

4. $3(\mathrm{H})$ \# $\mathrm{H}+\mathrm{H} H$

5. $4(+4)$ H H H H H

6. $5(H) \Rightarrow+H+H+$

7. $1(+) \quad+H+H+H$

8. $2(+)$ त $\mathrm{H}$ H H.H

9. $3(+)=\mathrm{H}+\mathrm{H}+\mathrm{H}$

10. $4(+), \quad+H+H+H$

11. $5(+),++H+H$

12. $6(+) \quad n+4+4$

13. $7(+) \quad++4 H+$

14. $8(+) \quad r+t+H$

15. $9(+) \quad * \quad+t+4$

16. $10(+)$ n +++++

17. $11(+) "+++++$

18. $1( \pm) ; \pm+4+4$

19. $2( \pm) \quad n+H+H$

20. $3( \pm)$ 土 + H H

21. $4( \pm) \quad n+++4$

22. $5( \pm) \quad n+ \pm \#+$

23. $6( \pm) \quad n+++1$

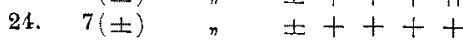

$\begin{array}{lllll}1^{\prime} & 2^{\prime} & 3^{\prime} & 4^{\prime} & 5^{\prime}\end{array}$

25. $8( \pm)$ stands for $\pm \pm+H+H$

26. $9( \pm) \quad n \quad \pm \pm+H+H$

27. $10( \pm)$ " $\pm \pm++H$

28. $11( \pm) \quad n \pm \pm++$

29. $12( \pm) \quad n \pm \pm \pm++$

30. $13( \pm) \quad$ \pm \pm \pm++

31. $14( \pm) \quad$ $\quad \pm \pm \pm \pm+$

32. $15( \pm) \quad$ " \pm \pm \pm \pm

33. $1(-), n \pm+H+H$

34. $2(-) \quad n- \pm+H+H$

35. $3(-) \quad \Rightarrow \quad \pm++H$

36. $4(-)$ " \pm+++

37. $5(-) \quad n- \pm \pm+4$

38. $6(-) \quad " \quad- \pm \pm++$

39. $7(-) \quad " \quad- \pm \pm \pm+$

40. $8(-) \quad " \quad- \pm \pm \pm \pm$

41. $9(-), \quad-- \pm++$

42. $10(-) \quad " \quad-\quad \pm++$

43. $11(-) \quad, \quad-- \pm \pm+$

44. $12(-) \quad n \quad-- \pm \pm \pm$

45. $13(-) \quad$ " $\quad- \pm \pm+$

46. $14(-)$ " - \pm \pm

47. $15(-) \quad, \quad---.- \pm$

48. $16(-) \quad \% \quad---1 \pm$

Explanation to the table:-

Take, for instance, the sign: $2(H)$. This stands for Arakawa's reaction with the course $(H) 1^{\prime}(H) 2^{\prime}(H) 3^{\prime}(H) 4^{\prime}(H) 5^{\prime}$. The sign does not express any prompt result of the reaction, so the prompt reaction of the sign : $2(+)$ may be $(-) 0^{\prime},( \pm) 0^{\prime}$, $(+) 0^{\prime}$ or even $(+) 0^{\prime}$, but this will not matter much, as the result of the reaction in one minute is the most important. 
0.840 and 0.808 grms. per 100 c.c. respectively on an average. Cf. Tables 5 and 6 ).

\section{TABle 5.}

Chlorine content (grms. per 100 c.c.) of mother's urine according A rakawa's reaction.

\begin{tabular}{|c|c|c|c|c|c|}
\hline Group & $\begin{array}{c}\text { Arakawa's reaction } \\
\text { R }=\text { right } \\
L=\text { left } \\
\end{array}$ & Case & Average & Maximum & Minimum \\
\hline 1 & $\begin{array}{l}\mathrm{R}(\#) 1^{\prime} \text { or } \mathrm{R} \text { or } \mathrm{L}(\#) \mathrm{I}^{\prime} \\
\mathrm{L}(\#) 1^{\prime} \text { or } \mathrm{L} \text { or } \mathrm{R}(\#) 1^{\prime} \\
\text { (Arakawa-positive case) }\end{array}$ & 14 & 1.062 & 1.355 & 0.725 \\
\hline 2 & $\begin{array}{l}\mathbf{R} \text { or } \mathrm{L}(+) \mathbf{I}^{\prime} \\
\mathbf{L} \text { or } \mathbf{R}(+) \mathbf{1}^{\prime}\end{array}$ & 5 & 0.882 & 1.172 & 0.753 \\
\hline 3 & $\begin{array}{l}\mathbf{R} \text { or } \mathbf{L}(+) \mathbf{I}^{\prime} \\
\mathbf{L} \text { or } \mathrm{R}( \pm) \mathbf{1}^{\prime}\end{array}$ & 5 & 0.893 & 0.994 & 0.817 \\
\hline 4 & $\begin{array}{l}\mathbf{R}(+) \mathbf{I}^{\prime} \\
\mathbf{L}(+) \mathbf{I}^{\prime}\end{array}$ & 18 & 0.990 & 1.318 & 0.511 \\
\hline 5 & $\begin{array}{l}\overline{\mathrm{R}} \text { or } \overline{\mathrm{L}}\left(+(+) \overline{1}^{\prime}\right. \\
\mathrm{L} \text { or } \mathrm{R}( \pm) \mathrm{I}^{\prime}\end{array}$ & 13 & 0.820 & 0.994 & 0.391 \\
\hline 6 & $\begin{array}{l}\mathbf{R} \text { or } \mathbf{L}(+) \mathbf{I}^{\prime} \\
\mathbf{L} \text { or } \mathrm{R}(-) \mathbf{I}^{\prime}\end{array}$ & 12 & 0.885 & 1.030 & 0.532 \\
\hline 7 & $\begin{array}{l}\mathrm{R}( \pm) \mathrm{I}^{\prime} \\
\mathrm{L}( \pm) \mathrm{I}^{\prime}\end{array}$ & 16 & 0.840 & 1.171 & 0.432 \\
\hline 8 & $\begin{array}{l}\mathbf{R} \text { or } \bar{L}( \pm) 1^{\prime} \\
\mathbf{L} \text { or } \mathbf{R}(-\{) \\
1^{\prime}\end{array}$ & 19 & 0.864 & 1.193 & 0.621 \\
\hline 9 & $\begin{array}{l}\mathrm{R}(-) \mathrm{I}^{\prime} \\
\mathrm{L}(-) \mathrm{I}^{\prime}\end{array}$ & 67 & 0.807 & 1.335 & 0.107 \\
\hline \multirow[t]{2}{*}{10} & $\begin{array}{l}\mathbf{R}(-) \mathbf{5}^{\prime} \\
\mathrm{L}(-) 5^{\prime} \\
\text { Completely Arakawa-negative }\end{array}$ & $\begin{array}{r}33 \\
\text { eases) } \\
\end{array}$ & 0.700 & 1.065 & 0.212 \\
\hline & Total & 202 & 0.874 & 1.355 & 0.107 \\
\hline
\end{tabular}

TABLE 6.

Chlorine content (grms. per 100 c.c.) of mothers' urine according to elinically positive and negative A rak aw a's reaction.

\begin{tabular}{c|c|c|c|c|c}
\hline & Arakawa's reaction & Case & Average & Maximum & Minimum \\
\hline \hline Arakawa-positive case & $($ H $) 1^{\prime}(+) 1^{\prime}$ & 14 & 1.062 & 1.355 & 0.725 \\
\hline $\begin{array}{c}\text { Weakly } \\
\text { Arakawa-negative case }\end{array}$ & $(+) 1^{\prime}( \pm) 1^{\prime}(-) I^{\prime}$ & 155 & 0.873 & 1.335 & 0.391 \\
\hline $\begin{array}{c}\text { Completely } \\
\text { Arakawa-negative case }\end{array}$ & $(-) 5^{\prime}$ & 33 & 0.700 & 1.065 & 0.212
\end{tabular}

The chlorine content of completely Arakawa-negative mothers shows an average figure $-34 \%$ deviation as compared with that of Arakawa-positive mothers (Cf. Table 7), and as to the maximum of chlorine content (Cf. Table 7), the deviation of the urine of completely Arakawa-negative mothers from that of Arakawa-positive mothers is 
TABle 7.

Chlorine content in urine of Arakawa-positive and completely negative mothers.

\begin{tabular}{c|c|c|c}
\hline & $\begin{array}{c}\text { Chlorine content in } \\
\text { urine of Arakawa- } \\
\text { positive mothers } \\
\text { (14 cases in number) }\end{array}$ & $\begin{array}{c}\text { Chlorine content in } \\
\text { urine of completely } \\
\text { Arakawa-negative } \\
\text { mothers } \\
\text { (33 cases in number) }\end{array}$ & $\begin{array}{c}\text { Deviation of } \\
\text { Arakawa-negative } \\
\text { cases from } \\
\text { positive eases }\end{array}$ \\
\hline $\begin{array}{c}\text { Average chlorine } \\
\text { content } \\
\text { (grms. per } 100 \text { c.c.) }\end{array}$ & 1.062 & 0.700 & $-34 \%$ \\
\hline $\begin{array}{c}\text { Maximum } \\
\text { (grms. per } 100 \text { c.c.) }\end{array}$ & 1.355 & 1.065 & $-21 \%$ \\
\hline $\begin{array}{c}\text { Minimum } \\
\text { (grms. per } 100 \text { c.c.) }\end{array}$ & 0.725 & 0.212 & $-70 \%$ \\
\hline
\end{tabular}

TABLE 8.

Distribution of all the samples according to A rak awa's reaction and to the amount of chlorine.

\begin{tabular}{|c|c|c|c|c|c|c|c|c|c|c|}
\hline $\begin{array}{l}\text { Group } \\
\text { Grms. } \\
\text { per } 100 \\
\text { c.c. }\end{array}$ & 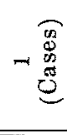 & 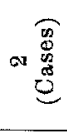 & 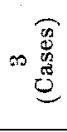 & 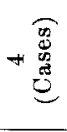 & 1. & 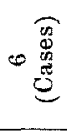 & 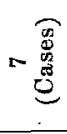 & 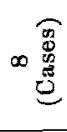 & 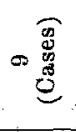 & 욜 \\
\hline 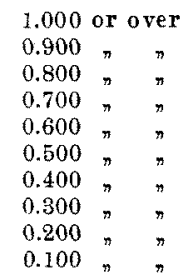 & $\begin{array}{l}7 \\
4 \\
2 \\
1\end{array}$ & $\begin{array}{l}2 \\
2\end{array}$ & $\begin{array}{l}2 \\
3\end{array}$ & $\begin{array}{l}7 \\
4 \\
5 \\
1 \\
1\end{array}$ & $\begin{array}{l}6 \\
2 \\
3 \\
1\end{array}$ & $\begin{array}{l}3 \\
3 \\
3 \\
2\end{array}$ & $\begin{array}{l}\text { I } \\
3 \\
6 \\
1 \\
3 \\
\\
1\end{array}$ & $\begin{array}{l}5 \\
2 \\
6 \\
4 \\
1 \\
1\end{array}$ & $\begin{array}{r}13 \\
9 \\
15 \\
8 \\
10 \\
5 \\
5 \\
1 \\
1\end{array}$ & $\begin{array}{l}3 \\
4 \\
5 \\
8 \\
1 \\
4 \\
5 \\
1 \\
2\end{array}$ \\
\hline
\end{tabular}

Table 9.

Distribution of all the samples according

to A rak awa's reaction and to the amount of chlorine.

\begin{tabular}{c|c|c|c}
\hline Groups & \\
Grms. & \\
per 100 \\
c.c.
\end{tabular}

léss remarkable $(-21 \%)$, while the minimum value of chlorine content deviation shows an average figure of $-70 \%$ (Cf. Table 7).

It will be thus seen that, in general, chlorine content of urine is larger in Arakawapositive mothers than Arakawanegative ones. One will notice the fact above mentioned from Table 8, too (Cf. Tables 8 and 9). 


\section{Literature and Comment.}

According to Is on $o,{ }^{4}$ the chlorine content in urine of healthy (Japanese) male adults is 0.871 grms. per 100 c.c. on an average. I cite here part of a table compiled by Is o no (Cf. Table 10).

TABLE 10.

Chlorine content of urine of healthy Japancse male adults.

\begin{tabular}{|c|c|c|c|c|c|c|c|c|}
\hline & Osawa) & Kaji ta) & $\begin{array}{c}\text { Huna- } \\
\text { oka }\end{array}$ & $\operatorname{Mori}{ }^{8}$ & In a b a & $\begin{array}{c}\mathrm{Yu}^{10} \\
\mathrm{kawa}\end{array}$ & satod a & Is on $\left.{ }^{12}\right)$ \\
\hline $\begin{array}{l}\text { Chlorine } \\
\text { (grms. per } \\
100 \text { c.c.) }\end{array}$ & 1.222 & 0.825 & 0.866 & 0.930 & 1.023 & 0.775 & 1.290 & 0.871 \\
\hline
\end{tabular}

The average chlorine content in all my cases, 202 in number, is 0.874 grms. per 100 c.c. on an average.

Now the Arakawa-negative milk is milk from a $*$ B-avitaminotic body, as has been shown by a number of works ${ }^{13-21)}$ published from our Department.

Omori, Ohashi, Nakanishi, Hara and Ota ${ }^{22)}$ fed their patients with slight affection of beriberi on B-avitaminotic diet, and found decrease of urine chlorine in the stage of exacerebation of the disease and an increase of it in the stage of convalescence. Then they investigated the feeding of healthy persons on B-avitaminotic diet

4) S. Is o no, Kaigun Gunikai Kaiho, $1913,5,76$.

5) O. Os a wa, Tokyo Igakkai Zasshi, 1889, 3, 667 .

6) K. Ka jita, Tokyo Igakkai Zasshi, 1890, 4, 275 and 1190.

7) E. Hun a ka, Tokyo Igakkai Zasshi, 1901, 15, 8.

8) R. Mori, Arbeiten aus der Kaiserl. Jap̉anischen militärarztlichen Lehranstalt, 1892, 1, 1 .

9) R. In a b a, Tokyo Igakkai"Zasshi, 1907, 21, 1025.

10) G. Y u k a wa, Tokyo Igakkai Zasshi, 1909, 23, 259,

11) G. Satod a, Tokyo Igakkai Zasshi, 1909, 23, 1143; 1910, 24, 411 and 991.

12) S. Is on o, Kaigun Gunikai Kaiho, 1913, 5, 76.

* In the present paper vitamin $B$ means vitamin $B_{1}$.

13) T. Arakawa, Tohoku J. Exp. Med, 1930, 16, 83 and 118.

14) A. Sato, Jikken Iho, 1983, 19, 1554.

15) A. Sato, Jikken Iho, 1985, 21, 523.

16) K. A sa kura and H. Os a ko, Tohoku J. Exp. Med., 1933, 20, 429.

17) T. Suzuki, Tohoku J. Exp. Med., 1934, 23, 28.

18) A. Tak a mats u, Tohoku J. Exp. Med., 1934, 23, 46.

19) A. Takamatsu, and A. Sato, Tohoku J. Exp. Med., 1934, 23, 506.

20) J. Kimura, Tohoku J. Exp. Med., 1934, 23, 494.

21) A. Tak a matsu, Tohoku J. Exp. Med., 1935, 27, 439.

22) K. Omori, Y. Ohashi, T. Nakanishi, M. Hara and T. Ota, Keio Igaku, $1923,3,197$. 
and found a decrease of urine chlorine.

Uehara, So and Fujinam $i^{23)}$ made an investigation into chlorine metabolism in beriberi and $B$-avitaminosis. An administration of vitamin $B$ in avitaminotic cases with edema would, they asserted, result in the excretion of $3 \sim 4$ times as much as chlorine in the urine as taken in. Miura ${ }^{24)}$ and $\mathrm{Hasui^{25 }}$ also found a decrease of urine chlorine in the stage of exacerebation of beriberi.

These results above cited are generally in conformity with the result of my own experiment (Tables 6 and 7 ). The abundance of chlorine (1.062 grms. per $100^{\circ}$ c.c. in Table 7) in the urine of Arakawa-positive mothers and the paucity of that of Arakawa-negative mothers (0.700 grms. per 100 c.c. in Table 7$)$ are very interesting facts. Even the minimum chlorine content in the former is larger $(0.725$ grms. in Table 7) than the average figure of 0.70 grms. in the latter. We know that Arakawa-negative milk is generally a milk from a Bavitaminotic body and a "bad" milk. One can easily conceive the existence of a close relation between Arakawa's reaction and the chlorine content of urine. Now with the result shown above we shall safely be able to conclude that the Arakawa-negative milk may pass for a B-avitaminotic milk also from the chlorine point of view.

Now in $1934 \mathrm{Nozaki}^{26)}$ concluded that human milk negative to Arakawa's reaction was essentially higher in chlorine content than the Arakawa-positive milk. In 1938, Is hii ${ }^{26)}$ (Cf. 95th Report of the Peroxidase Reaction) reported that chlorine content in the blood of mothers with negative A rakawa's reaction is slightly richer than in that of mothers with positive Arakaw a's reaction.

Considering solely the chlorine content of milk, and of urine, the Arakawa-negative mother is rich in milk chlorine and poor in urine chlorine. So there is highly probably a tendency to chlorine retention in an Arakawa-negative mother.

\section{Summary.}

Human milk negative to Arakawa's reaction is generally milk from a B-avitaminotic body as shown by a number of papers from our

\footnotetext{
* "bad" milk:-generally such a human milk with weak or negative Arakawa's reaction.

23) I. Uehara, T. So and H. Fujin a mi, Tokyo Igakkai Zasshi, 1922, 36, 1421.

24) K. Mi u ra, Ergeb. d. inn. Med. n. Kinderhk., 1909, 4, 280.

25) N. Hasui, Nippon Naikagakkai Zasshi, 1922, 10, 169.

26) J. Noz a ki, Tohoku J. Exp. Med., 1934, 23, 60.
} 
Laboratory. Viewed from the chlorine content of human urine, the Arakawa-negative mother shows an essentially lower content. And it is evident from the above cited literature that a B-avitaminotic body shows lower content of chlorine in urine. It is very probable that, viewed from the chlorine content of mother's urine, the Arakawanegative human milk will pass for a B-avitaminotic milk even to the exclusion of other possibilities.

\section{Conclusion.}

1. Urine of Arakawa-negative mothers is essentially lower in chlorine content than that of Arakawa-positive mothers.

2. Chlorine content in the urine of Arakawa-negative mothers shows an average figure of $-34 \%$ deviation as compared with that of Arakawa-positive mothers.

3. Viewed from the chlorine content of milk (Nozaki and Ishii), blood (Ishii) and urine (author), it is not difficult to presume that there is a tendency to abnormal chlorine retention in Arakawanegative mothers.

27) M. Is hii, Tohoku J. Exp. Med., 1988, 33, 71. 